\title{
Constant pressure hybrid Molecular Dynamics-Monte Carlo simulations
}

\author{
Roland Faller and Juan J. de Pablo ${ }^{\text {a) }}$ \\ Department of Chemical Engineering, University of Wisconsin, Madison, Wisconsin 53706
}

(Received 3 August 2001; accepted 28 September 2001)

\begin{abstract}
New hybrid Molecular Dynamics-Monte Carlo methods are proposed to increase the efficiency of constant-pressure simulations. Two variations of the isobaric Molecular Dynamics component of the algorithms are considered. In the first, we use the extended-ensemble method of Andersen [H. C. Andersen, J. Chem. Phys. 72, 2384 (1980)]. In the second, we arrive at a new constant-pressure Monte Carlo technique based on the reversible generalization of the weak-coupling barostat [H. J. C. Berendsen et al., J. Chem. Phys. 81, 3684 (1984)]. This latter technique turns out to be highly effective in equilibrating and maintaining a target pressure. It is superior to the extended-ensemble method, which in turn is superior to simple volume-rescaling algorithms. The efficiency of the proposed methods is demonstrated by studying two systems. The first is a simple Lennard-Jones fluid. The second is a mixture of polyethylene chains of 200 monomers. (C) 2002 American Institute of Physics. [DOI: 10.1063/1.1420460]
\end{abstract}

\section{INTRODUCTION}

Molecular simulations at constant pressure are attractive because they mimic the conditions often encountered in laboratory experiments. This applies to both Molecular Dynamics and Monte Carlo calculations. In Monte Carlo simulations, trial configurations are generated in a random manner, and accepted according to well-defined probability criteria. The so-called "hybrid" schemes constitute one of many ways of proposing such trial configurations. In a hybrid Monte Carlo trial move, a short molecular dynamics trajectory is used to generate a new configuration; ${ }^{1,2}$ the move is subsequently accepted or rejected according to probability criteria which take into account the way in which the trial configuration was generated (since molecules are moved in the direction of the forces acting on them, the resulting bias must be eliminated). Molecular Dynamics becomes exact in the limit of a vanishing time-step; the acceptance rate of trial configurations produced by a short MD run with small time-steps can therefore be expected to be high. The use of longer time-steps does not deteriorate the accuracy of a hybrid scheme, but it decreases the acceptance rate. The Monte Carlo acceptance/rejection procedure ensures that the correct statistical-mechanical ensemble is sampled, and the results remain exact, regardless of the time-step. In contrast, a pure molecular dynamics simulation using large time-steps would lead to a fast algorithm but questionable results.

Many algorithms for isobaric-isothermal NPT molecular dynamics have been developed over the past decades. ${ }^{3-6}$ In contrast, NPT Monte Carlo simulations still rely on the use of simple, random volume changes accompanied by uniform rescaling of the molecules' coordinates. A few exceptions have been reported in the literature, but they lack the ease of implementation of simple random volume moves. ${ }^{7}$ Unfortunately, volume relaxation is notoriously slow in simple, random volume-rescaling Monte Carlo algorithms.

a)Electronic mail: depablo@engr.wisc.edu
Furthermore, their efficiency deteriorates as the size of a system increases.

Our experience indicates that volume moves are actually one of the limiting steps towards successful Monte Carlo simulations of complex fluids. It has, for example, become clear in studies of solubilities of small molecules in polymers that the computational bottleneck is indeed the equilibration of the density. ${ }^{8}$ Interestingly, literature results suggest that constant-pressure molecular dynamics algorithms can provide relatively fast volume relaxation. It is therefore of interest to explore whether a hybrid NPT method is able to circumvent some of the problems associated with conventional NPT Monte Carlo techniques. In this paper we consider two hybrid NPT approaches. We find both of these to be superior to conventional methods for constant-pressure Monte Carlo simulations.

\section{CONSTANT PRESSURE MONTE CARLO}

Before presenting the two hybrid methods to be discussed here, we shortly review the fundamentals of Monte Carlo simulations in the isothermal-isobaric ensemble. The natural variables are particle number $N$, pressure $P$, and temperature $T$; the statistical mechanical potential for this set of variables is the Gibbs free energy $G(N, P, T)$. As $N, P$ and $T$ are fixed, their conjugate variables must fluctuate. These are the chemical potential $\mu$ (which, for a pure system, is equal to the Gibbs free energy density $G / N$ ), the system volume $V$ and the entropy $S$.

The relevant probability distribution function for an NPT ensemble is given by

$$
f_{\mathrm{NPT}}=\exp \left[-\beta U(\{\vec{r}\},\{\vec{p}\})-\beta P^{*} V(\{\vec{r}\})\right] .
$$

Here $P^{*}$ is the system pressure, $\{\vec{p}\}$ is the set of all momenta, $\{\vec{r}\}$ the set of all positions, $U$ the internal energy of the system and $V$ its volume; $\beta=1 /\left(k_{\mathrm{B}} T\right)$, where $k_{\mathrm{B}}$ is Boltzmann's constant and $T$ is the temperature. 
This distribution function is to be sampled by a simulation in an NPT ensemble; the following acceptance criteria for trial moves generate configurations distributed according to Eq. (1):

$$
p_{\text {acc }}=\min \left\{1, \exp \left[-\beta \Delta U\left(\{\vec{r},\{\vec{p}\})-\beta P^{*} \Delta V(\{\vec{r}\})\right]\right\},\right.
$$

where $\Delta$ denotes the difference of a property between the new (proposed) state and the old (original) state, and where $\min (x, y)$ denotes the minimum of its two arguments. The condition of detailed balance, namely

$$
\begin{aligned}
& K(a \rightarrow b)=K(b \rightarrow a), \\
& K(a \rightarrow b)=f(a) q(a \rightarrow b) p_{\text {acc }}(a \rightarrow b),
\end{aligned}
$$

facilitates the construction of a correct algorithm. In Eq. (3), $K(a \rightarrow b)$ is the probability of going from state $a$ to state $b$; this probability can be written as the product of $f(a)$, the probability of being in state $a, q(a \rightarrow b)$, the probability of proposing a transition from $a$ to $b$, and $p_{\text {acc }}(a \rightarrow b)$, the probability of accepting such a transition. Accepting or rejecting trial moves according to

$$
p_{\text {acc }}(a \rightarrow b)=\min \left(1, \frac{f(b) q(b \rightarrow a)}{f(a) q(a \rightarrow b)}\right)
$$

ensures that configurations are distributed according to the desired probability distribution $f$ appearing in Eq. (3). For an isothermal-isobaric (NPT) ensemble, $f$ is given by Eq. (1).

\section{THE EXTENDED ENSEMBLE ALGORITHM}

In order for a Molecular Dynamics algorithm to be suitable for direct application within a hybrid Monte Carlo formalism, two conditions must be fulfilled. The algorithm should be reversible in time and symplectic, i.e., preserving phase-space volume. If these requirements are met, Eq. (3) is fulfilled and the correct Markov process is produced. Given these constraints, the Verlet integration scheme has often been used in this context. ${ }^{9}$ It is reversible, it preserves phasespace volume and it is correct to order $O\left(\Delta t^{3}\right)$.

The extended-ensemble technique of Andersen ${ }^{3}$ (in conjunction with the Verlet integrator) satisfies the above prerequisites. Its application in the context of a hybrid move is therefore promising. The relevant equations of motion are given by (for a derivation see the original paper of Andersen $\left.^{3}\right)$ :

$$
\begin{aligned}
& \mathrm{d}_{t} \vec{r}_{i}=\frac{p_{i}}{m_{i}}+\frac{1}{3} \mathrm{~d}_{t} \ln V, \\
& \mathrm{~d}_{t} \vec{p}_{i}=-\sum_{j^{\prime}=1}^{N} \hat{r}_{i j} u^{\prime}\left(r_{i j}\right), \\
& M \mathrm{~d}_{t}^{2} V=-\alpha+\frac{1}{V}\left(\frac{2}{3} \sum_{i=1}^{N} \frac{p_{i}^{2}}{2 m}-\frac{1}{3} \sum_{i<j=1}^{N} r_{i j} u^{\prime}\left(r_{i j}\right)\right),
\end{aligned}
$$

where $M$ is a fictitious mass of a "piston" associated with the volume move (the piston connects the system to an infinitely large pressure reservoir). Parameter $\alpha$ describes the fictitious potential of the volume. These equations can be easily implemented, and the resulting molecular dynamics trajectory obeys a constant NPH constraint. Anderson's method can be generalized to constant NPT, but this is not of interest here as maintaining a constant temperature is left to the Monte Carlo acceptance criteria.

In most Monte Carlo simulations one deals with particle positions only. Therefore, at the beginning of a hybrid step, velocities (or momenta) must be assigned to each particle. These are generated at random from a Maxwell-Boltzmann distribution (i.e., with probability proportional to the exponential of the kinetic energy). In addition, a "volume" velocity $v_{V}$ is also generated.

The acceptance criteria for a volume hybrid move are derived from Eqs. (3) and (4). Since the integration of the equations of motion itself is deterministic, after having assigned velocities to the molecules, the probability of reaching a final state from some original state is unity. Still, the probability of assigning velocities to the particles must be considered, thereby leading to transition probabilities of the form

$$
\begin{aligned}
& q(a \rightarrow b)=\exp \left(E_{\mathrm{kin}}^{(\mathrm{a})}\right) f\left(v_{V}^{(\mathrm{a})}\right), \\
& q(b \rightarrow a)=\exp \left(E_{\mathrm{kin}}^{(\mathrm{b})}\right) f\left(v_{V}^{(\mathrm{b})}\right),
\end{aligned}
$$

where $f\left(v_{V}\right)$ is the probability of generating a volume velocity according to a specific distribution, $E_{\text {kin }}$ is the kinetic energy and superscripts $a$ and $b$ serve to denote the original and new (trial) states, respectively. The acceptance criteria for hybrid NPT moves are obtained by substituting Eqs. (6) into Eq. (4).

\section{THE REVERSIBLE WEAK-COUPLING ALGORITHM}

One of the most efficient algorithms for constant temperature and/or pressure Molecular Dynamics is the weakcoupling scheme proposed by Berendsen et al. ${ }^{5}$ Although it is widely used, unlike Andersen's algorithm, it is not timereversible due to the first-order nature of the equations of motion. This precludes its direct use in hybrid Monte Carlo moves. The algorithm proposed in what follows eliminates this shortcoming.

Recently, it has been shown that the weak-coupling thermostat produces a correct ensemble, in agreement with other thermostat methods for any value of the coupling parameter $\tau .{ }^{10}$ We briefly describe the weak-coupling algorithm, focusing only on the constant-pressure case. In any Molecular Dynamics algorithm the integrator moves the velocities and positions in a time-step according to the equations of motion:

$$
\begin{aligned}
& \vec{r}_{i}(t) \rightarrow \vec{r}_{i}(t+\Delta t), \\
& \vec{v}_{i}(t) \rightarrow \vec{v}_{i}(t+\Delta t) .
\end{aligned}
$$

After this update, the weak-coupling algorithm calculates the new pressure $P$ of the system based on the positions and velocities. This pressure is compared to the desired, target pressure $P^{*}$. The volume of the simulation box is now scaled "towards" the target pressure, i.e., 


$$
V(t+\Delta t)=\tau_{p}^{-1} V(t) \cdot\left(\frac{P(t)-P^{*}}{P^{*}}\right) \Delta t,
$$

where $\tau_{p}$ is a characteristic time for this relaxation process (see below), and $\Delta t$ is usually (although not necessarily) the same time-step used to integrate the equations of motion. The positions of all particles must be adapted to the new volume, i.e., rescaled by a factor $V^{1 / 3}$. For convenience and efficiency, the molecular dynamics step is performed in scaled coordinates ranging from $s=r / V^{1 / 3}=[-0.5,0.5]$.

The smaller the relaxation time $\tau_{p}$, the more closely the instantaneous pressure is tied to the target, and the stronger the disturbance of the actual dynamics by individual rescaling operations. In molecular dynamics, $\tau_{p}$ must be large enough to produce meaningful data, as the fluctuations of pressure produced by this algorithm are not entirely correct. ${ }^{11}$ This does not pose a problem for Monte Carlo, as the molecular dynamics moves are only used to propose trial configurations.

As pointed out earlier, the volume rescaling of the weakcoupling algorithm is not time-reversible. This violates the detailed balance rate equation (3). The solution to this problem is to allow the algorithm to run explicitly backward and forward in time; at the beginning of a hybrid move, a random number $\zeta$ is drawn between 0 and 1 . If $\zeta<0.5$, the time direction in the simulation runs forward; otherwise we run backwards. Running the simulation in reverse is equivalent to setting the relaxation time $\tau_{p}$ to a negative value, as the rest of the equations of motion is symmetric under timereversal. The acceptance criteria for this constant pressure hybrid move is similar to that for the Andersen case.

The weak-coupling method as outlined so far results in rather slow fluctuations around the desired pressure. Furthermore (if backward moves are momentarily disregarded), the relaxation becomes exponentially slow close to the target pressure. This limits the efficiency of the simulation. To improve efficiency, we generalize the weak-coupling hybrid scheme to a target pressure selected randomly within a bounded domain. The pressure $P^{*}$ used as local target pressure for the simulation in one hybrid step is now chosen with uniform probability from the interval $\left[\left(1-P_{\text {range }}\right) P^{(\text {ext })}\right.$, $\left(1+P_{\text {range }}\right) P^{(\text {ext })}$, whereas the pressure in the acceptance criteria remains untouched (at $P^{(\mathrm{ext})}$ ). Values for $P_{\text {range }}$ range from 0 to 0.1 . Since the local target pressure is uniformly chosen from the neighborhood of the global target pressure, detailed balance is still obeyed.

\section{EFFICIENCY CONSIDERATIONS}

The main parameter which can be tuned and optimized in the reversible weak-coupling (RWC) simulation is the relaxation time $\tau_{p}$. The smaller $\tau_{p}$, the closer the system follows the desired target pressure, but the lower is the acceptance rate.

We analyze the density autocorrelation function to provide a measure of efficiency for the proposed hybrid schemes. Figure 1(a) shows that, for a simple Lennard Jones fluid, increasing the time-step leads to smaller correlation times and faster equilibration of the density. However, if the
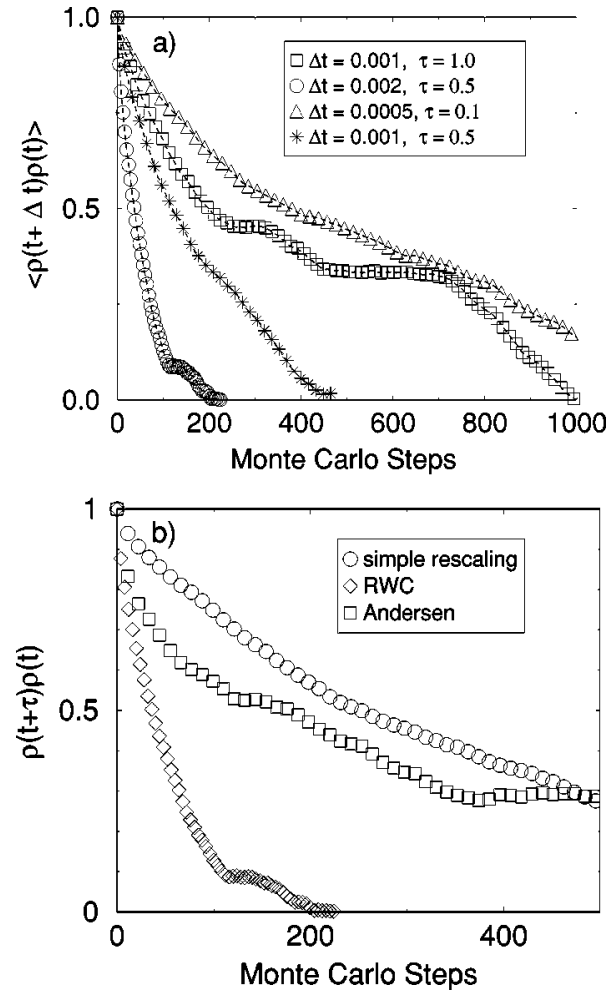

FIG. 1. Density autocorrelation function for a Lennard-Jones fluid ( $T$ $=1.4, p=0.21732, \rightarrow \rho=0.45)$ with different values of time-step and correlation time $\left[\right.$ part (a)]. $p_{\text {range }}=0.1$ the values for the timestep and the correlation time are given in the graph. Part (b) shows the comparison to simple rescaling and the hybrid method based on the Andersen barostat. The box mass $M$ in the Andersen case was set to $M=1.0$.

ratio of correlation time to time-step becomes too small the simulation becomes again inefficient as one would need extremely large neighbor lists (or extremely frequent updates) to balance the rapid motion. The figure shows how important a good choice of the parameters can be. An unfortunate choice results in only marginal improvements relative to conventional methods.

The ratio between the time-step and the correlation time is important. The correlation function for $\Delta t=0.001, \tau=0.25$ is the same as for $\Delta t=0.002, \tau=0.5$ (not shown). We found that a value of this ratio of about 250 provides good efficiencies. If we increase this ratio the acceptance rate drops; if we decrease it the efficiency of the single move deteriorates. The conventional method for constant pressure Monte Carlo simulations comprises simple volume-rescaling trial moves. In such moves a random change of volume is proposed according to

$$
V_{\text {new }}=V_{\text {old }}+\Delta V \text {, }
$$

with $\Delta V$ chosen uniformly from $\left[-\Delta V_{\max }, \Delta V_{\max }\right]$.

Our results indicate that, even for a simple LennardJones fluid, the hybrid techniques proposed here provide methods that appear to be superior to the simple volumerescaling technique. For a good choice of parameters, the efficiency gains can amount to more than an order of magnitude. 
TABLE I. A comparison of density and internal energy per particle for the Lennard-Jones system from simulation of this work using reversible weak coupling (1) and from the work of Johnson et al. (2).

\begin{tabular}{clcccc}
\hline \hline $\mathrm{T}$ & \multicolumn{1}{c}{$\mathrm{p}$} & $\rho_{1}$ & $u_{1}$ & $\rho_{2}$ & $u_{2}$ \\
\hline 0.75 & 1.59 & $0.90 \pm 0.01$ & $-6.35 \pm 0.03$ & 0.904 & -6.40 \\
1.6 & 0.1231 & $0.096 \pm 0.002$ & $-0.73 \pm 0.03$ & 0.094 & -0.67 \\
1.6 & 0.16 & $0.132 \pm 0.001$ & $-0.91 \pm 0.02$ & 0.132 & -0.91 \\
1.6 & 0.38 & $0.42 \pm 0.01$ & $-2.79 \pm 0.03$ & 0.408 & -2.72 \\
1.6 & 5.07 & $0.84 \pm 0.01$ & $-5.10 \pm 0.03$ & 0.85 & -5.27 \\
\hline \hline
\end{tabular}

\section{TEST CASES}

\section{A. The Lennard-Jones fluid}

As a test case, the Lennard-Jones fluid is revisited. We simulate a system of 200 Lennard-Jones particles at a range of temperature and pressures. The cutoff is $r=2.5 \sigma$ and the usual analytic long-range corrections for energy and pressure are applied. ${ }^{12}$ All values are given in the standard dimensionless units. The simulations used 1 million MC steps, each containing 5 constant-pressure molecular dynamics integration steps.

We compare our results to those obtained from an equation of state ${ }^{13}$ which is known to provide accurate properties for the Lennard-Jones fluid. At a given temperature and pressure we compare density and internal energy. The results are shown in Table I; an agreement between simulations and equation of state predictions is excellent, showing that the algorithms proposed here lead to correct property predictions. Note that we simulated the same systems (using identical starting conditions) using the three methods considered in this work (Andersen, Weak-Coupling and Simple VolumeRescaling). There were no noticeable differences in the results.

We now examine the volume fluctuations produced by the different methods. These fluctuations are of interest because they provide a stringent test of the correctness and the efficiency of a simulation technique, and they are related to the compressibility of the fluid. We compare our results to the equation of state by Rosenfeld, ${ }^{14}$ again at $T=1.6$ (cf. Table II). As expected, the compressibilities corresponding to our various simulation techniques agree with accepted values,

$$
\kappa_{T}=\frac{\left\langle V^{2}\right\rangle-\langle V\rangle^{2}}{k_{\mathrm{B}} T V} .
$$

TABLE II. Isothermal compressibilities for the Lennard-Jones fluid at $T$ $=1.6$ calculated by fluctuations of the volume $\left(\kappa_{T 1}\right)$ and by the equation of state by Rosenfeld $\left(\kappa_{T 2}\right)$.

\begin{tabular}{cll}
\hline \hline$\rho$ & \multicolumn{1}{c}{$\kappa_{T 1}$} & $\kappa_{T 2}$ \\
\hline 0.13 & $6.7 \pm 2$ & $\ldots$ \\
0.40 & $2.4 \pm 0.5$ & 2 \\
0.84 & $0.04 \pm 0.01$ & 0.05 \\
\hline \hline
\end{tabular}
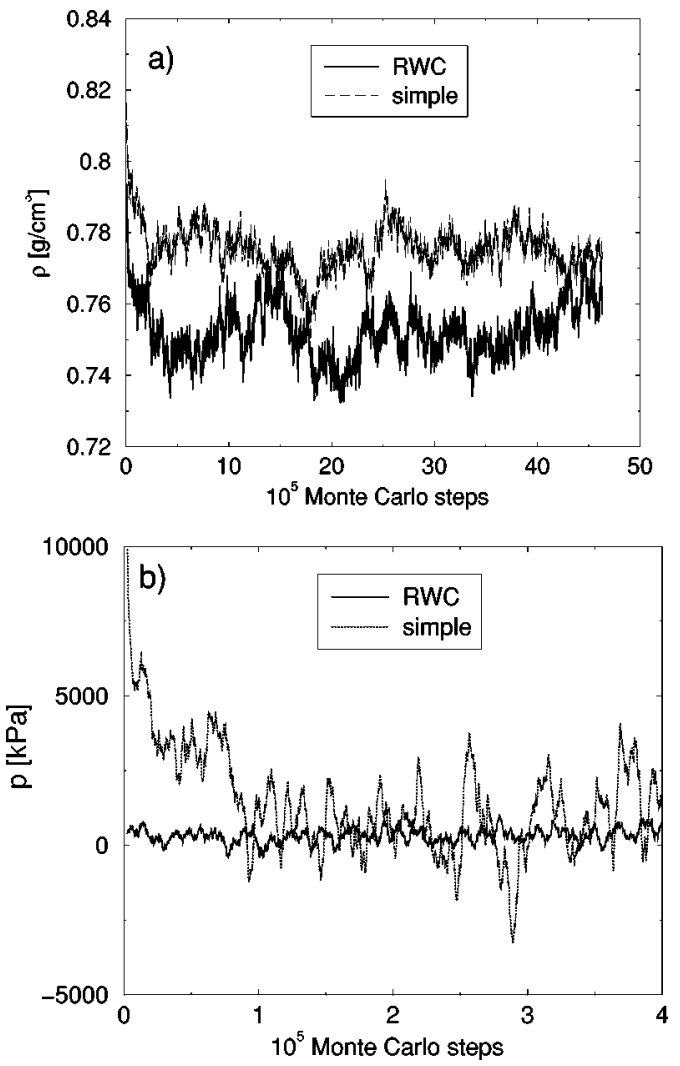

FIG. 2. (a) Density and (b) pressure as a function of simulation time for the reversible Weak-Coupling algorithm an the volume-rescaling algorithm for a system of 11 alkane chains of length 200 and 26 ethylene molecules at $P$ $=260 \mathrm{kPa}, T=400 \mathrm{~K}$. For the pressure, a running average over 50 data points was performed in order to suppress the high frequency spikes and improve clarity.

\section{B. Polymer melts}

In order to examine the performance of the proposed hybrid methods in the context of a complex fluid, we used them to equilibrate a polymer melt.

Systems consisting of linear polyethylene chains $\left(\mathrm{C}_{100}\right.$ to $\mathrm{C}_{500}$ ) and a small fraction of ethylene were placed in a simulation box at a density above the experimental value. Three identical systems were left to equilibrate by the reversible weak-coupling hybrid methods and the volume-rescaling algorithm. The weak-coupling algorithm proved to be efficient and produced reliable, well-equilibrated densities for the pressure range under study $(200-500 \mathrm{kPa})$. All studies have been performed using the NERD force-field. ${ }^{8,15}$

Figure 2 shows a comparison between the convergence of the newly proposed weak-coupling method and simple volume rescaling. Both simulations started at a system density of $0.82 \mathrm{~g} / \mathrm{cm}^{3}$, which is slightly too high. The equilibrium density is around $0.75 \mathrm{~g} / \mathrm{cm}^{3}$. It can be seen in the figure that the convergence of the weak-coupling algorithm to the correct, target pressure is relatively fast. In contrast, pressure (or volume) relaxation in the conventional volumerescaling simulation is slow. Even after 500,000 steps, the running-average pressure in the conventional volumerescaling simulation is far from the correct, target result (by about a factor of 3 ). 


\section{CONCLUSIONS}

New hybrid schemes have been presented for NPT Monte Carlo simulations. We find that the proposed methods are more effective at relaxing the volume of simple and complex liquids than conventional Monte Carlo techniques. The increase of efficiency becomes more apparent in fluids that exhibit long relaxation times (e.g., polymers).

It is our experience that attaining the equilibrium density of a dense polymer melt using conventional NPT Monte Carlo moves is highly computationally demanding, and often not possible at all. In fact, we have shown in this work that as a result of poor sampling, the compressibility of a melt of short polyethylene chains determined using simple rescaling techniques deviates considerably from the correct value. In contrast, the compressibility predicted using the weakcoupling algorithm is in agreement with experimental values.

An additional advantage of the methodologies discussed in this work is that available simulation codes for molecular dynamics calculations can be modified to perform exact Monte Carlo calculations with minimal changes. Implementation of the proposed techniques does not add a significant overhead, and the new moves not only change the volume, but also contribute significantly to the repositioning of particles, thereby improving sampling.

\section{ACKNOWLEDGMENTS}

Discussions with Brian Banaszak and Kevin van Workum are gratefully acknowledged. One of the authors (R.F.) thanks the DFG (German Research Foundation) for financial support in the framework of the Emmy-Noether program. This work was supported by the Division of Chemical Sciences, Office of Basic Energy Sciences, U.S. Department of Energy. Acknowledgment is also made to the Donors of the Petroleum Research Fund, administered by the ACS, for partial support of this research.

${ }^{1}$ D. Heermann, P. Nielaba, and M. Rovere, Comput. Phys. Commun. 60, 311 (1990).

${ }^{2}$ B. Mehlig, D. W. Heermann, and B. M. Forrest, Phys. Rev. B 45, 679 (1992).

${ }^{3}$ H. C. Andersen, J. Chem. Phys. 72, 2384 (1980).

${ }^{4}$ M. Parinello and A. Rahman, J. Chem. Phys. 76, 2662 (1983).

${ }^{5}$ H. J. C. Berendsen, J. P. M. Postma, W. F. van Gunsteren, A. DiNola, and J. R. Haak, J. Chem. Phys. 81, 3684 (1984).

${ }^{6}$ G. J. Martyna, M. E. Tuckerman, D. J. Tobias, and M. L. Klein, Mol. Phys. 87, 1117 (1996)

${ }^{7}$ F. A. Escobedo and J. J. de Pablo, Macromol. Theory Simul. 4, 691 (1995)

${ }^{8}$ S. K. Nath and J. J. de Pablo, Mol. Phys. 98, 231 (2000).

${ }^{9}$ L. Verlet, Phys. Rev. 159, 98 (1967).

${ }^{10}$ T. Morishita, J. Chem. Phys. 113, 2976 (2000).

${ }^{11}$ E. Paci and M. Marchi, J. Phys. Chem. 100, 4314 (1996).

${ }^{12}$ M. P. Allen and D. J. Tildesley, Computer Simulation of Liquids (Clarendon, Oxford, 1987)

${ }^{13}$ J. K. Johnson, J. A. Zollweg, and K. E. Gubbins, Mol. Phys. 78, 591 (1993).

${ }^{14}$ Y. Rosenfeld, Mol. Phys. 94, 929 (1998).

${ }^{15}$ S. K. Nath, B. J. Banaszak, and J. J. dePablo, J. Chem. Phys. 114, 3612 (2001). 